\title{
Four-Qubit Device with Mixed Couplings
}

\author{
M. Grajcar, ${ }^{1,2,3}$ A. Izmalkov, ${ }^{1}$ S. H. W. van der Ploeg, ${ }^{1,4}$ S. Linzen, ${ }^{1}$ T. Plecenik,${ }^{1,2}$ Th. Wagner,,${ }^{1}$ U. Hübner, ${ }^{1}$ E. Il'ichev, ${ }^{1, *}$ \\ H.-G. Meyer, ${ }^{1}$ A. Yu. Smirnov, ${ }^{5}$ Peter J. Love, ${ }^{5}$ Alec Maassen van den Brink, ${ }^{5, \dagger}$ \\ M.H. S. Amin, ${ }^{5}$ S. Uchaikin, ${ }^{5}$ and A. M. Zagoskin ${ }^{6, *}$ \\ ${ }^{1}$ Institute for Physical High Technology, P.O. Box 100239, D-07702 Jena, Germany \\ ${ }^{2}$ Department of Solid State Physics, Comenius University, SK-84248 Bratislava, Slovakia \\ ${ }^{3}$ Center of Excellence of the Slovak Academy of Sciences (CENG), Slovakia \\ ${ }^{4} \mathrm{MESA}+$ Research Institute and Faculty of Science and Technology, University of Twente, \\ P.O. Box 217, 7500 AE Enschede, The Netherlands \\ ${ }^{5}$ D-Wave Systems Inc., 100-4401 Still Creek Drive, Burnaby, British Columbia, V5C 6G9 Canada \\ ${ }^{6}$ Physics and Astronomy Dept., The University of British Columbia, \\ 6224 Agricultural Road, Vancouver, British Columbia, V6T 1 Z1 Canada
}

(Received 21 September 2005; published 2 February 2006)

\begin{abstract}
We present the first experimental results on a device with more than two superconducting qubits. The circuit consists of four three-junction flux qubits, with simultaneous ferro- and antiferromagnetic coupling implemented using shared Josephson junctions. Its response, which is dominated by the ground state, is characterized using low-frequency impedance measurement with a superconducting tank circuit coupled to the qubits. The results are found to be in excellent agreement with the quantum-mechanical predictions.
\end{abstract}

DOI: 10.1103/PhysRevLett.96.047006

The implementation of one- and two-qubit gates in superconducting qubit prototypes [1] has confirmed their utility for quantum computation. Such qubits are readily fabricated, and highly scalable in principle. A prominent subcategory consists of superconducting loops interrupted by three Josephson junctions, so-called 3JJ flux qubits [2]. If threaded by a near-degenerate magnetic bias flux $\Phi^{\mathrm{x}} \approx$ $\frac{1}{2} \Phi_{0}\left(\Phi_{0}\right.$ is the flux quantum), such devices have quantum states which are superpositions of clockwise $(|\downarrow\rangle)$ and counterclockwise $(|\uparrow\rangle)$ circulating supercurrent $I_{\mathrm{p}}$. The small area of 3JJ qubits reduces their coupling to environmental magnetic noise, making comparatively long coherence times possible [3], but also limits the strength of their inductive coupling $[2,4]$. This can be overcome using direct galvanic coupling through a shared Josephson junction [5]. When reporting its experimental realization [6], we mentioned that direct coupling can have additional advantages. First, the coupling strength can be varied independently of the sample geometry by changing the shared junction's critical current. Second, while direct coupling normally has the antiferromagnetic (AFM) sign just as in the inductive case, a "twisted" design [joining two qubit loops in a " $\infty$ " shape with crossing leads in the center; see Fig. 1(b)] features ferromagnetic (FM) coupling [7].

In this Letter, we pursue this by studying a four-qubit circuit in which the two types of coupling coexist. This is very promising from the perspectives of realizing nontrivial Ising-spin systems [8] and scalable adiabatic quantum computing (AQC) $[9,10]$. However, while our circuit behaves in full agreement with quantum mechanics and features excellent multiqubit bias control, the measurements presented are essentially equilibrium. The system's effective low-energy (pseudospin) Hamiltonian is
PACS numbers: 85.25.Dq, 03.67.Lx, 85.25.Cp

$$
H=-\sum_{i=1}^{4}\left[\epsilon_{i} \sigma_{z}^{(i)}+\Delta_{i} \sigma_{x}^{(i)}\right]+\sum_{1 \leq i<j \leq 4} J_{i j} \sigma_{z}^{(i)} \sigma_{z}^{(j)},
$$

where $\epsilon_{i}$ is the bias on qubit $i$ (implemented through a flux bias $\left.\Phi^{\mathrm{x}}-\frac{1}{2} \Phi_{0}\right), \Delta_{i}$ is its tunneling amplitude, $J_{i j}$ is the coupling energy between qubits $i$ and $j$, and $\sigma_{z}, \sigma_{x}$ are Pauli matrices in the span of $|\downarrow\rangle\left(\sigma_{z}=-1\right)$ and $|\uparrow\rangle$ $\left(\sigma_{z}=1\right)$. Equation (1) adequately describes a system of flux qubits [2,4,11], although possible limitations [12] remain to be investigated.

Our approach follows earlier work using the impedance measurement technique (IMT) [13]. This method measures impedance shifts in a high-quality superconducting $L C$ (tank) circuit inductively coupled to the system. For one qubit [14], the average loop current $I=\left(|b|^{2}-|a|^{2}\right) I_{\mathrm{p}}$ in an eigenstate $a|\downarrow\rangle+b|\uparrow\rangle$ changes direction at the anticrossing point $\Phi^{\mathrm{x}}=\frac{1}{2} \Phi_{0}$ (where $|a|=|b|$, i.e., the pseudospin ground state flips from $|\downarrow\rangle$ to $|\uparrow\rangle$ and vice versa for the excited state). This causes a peak in the qubit susceptibility $\chi=d I / d \Phi^{\mathrm{x}}$; since $d I / d \Phi^{\mathrm{x}} \propto d^{2} E / d\left(\Phi^{\mathrm{x}}\right)^{2}$, where $E$ is the average energy and $d_{\Phi^{\mathrm{x}}}$ is adiabatic (note 15 in Ref. [14]), this also follows from the large energy-band curvature at the anticrossing. Note that the very presence of such peaks is already a quantum effect, since the classical $\chi\left(\Phi^{\mathrm{x}}\right)$ curves feature hysteretic loops [10] in the absence of flux tunneling. Because of the qubit-tank coupling, $\chi$ affects the tank's eigenfrequency $\omega_{\mathrm{T}}$ and hence, under resonant driving, its current-voltage phase angle $\Theta \propto \chi$. Thus, the width and height of such an IMT peak in $\Theta$ provide information about the anticrossing, i.e., about $\Delta$ and $I_{\mathrm{p}}$.

For multiple qubits, a more general analysis yields [15]

$$
\tan \Theta=-2 \frac{Q_{\mathrm{T}}}{L_{\mathrm{T}}} \sum_{\mu<\nu} \frac{\rho_{\mu}-\rho_{\nu}}{E_{\nu}-E_{\mu}} R_{\mu \nu}
$$



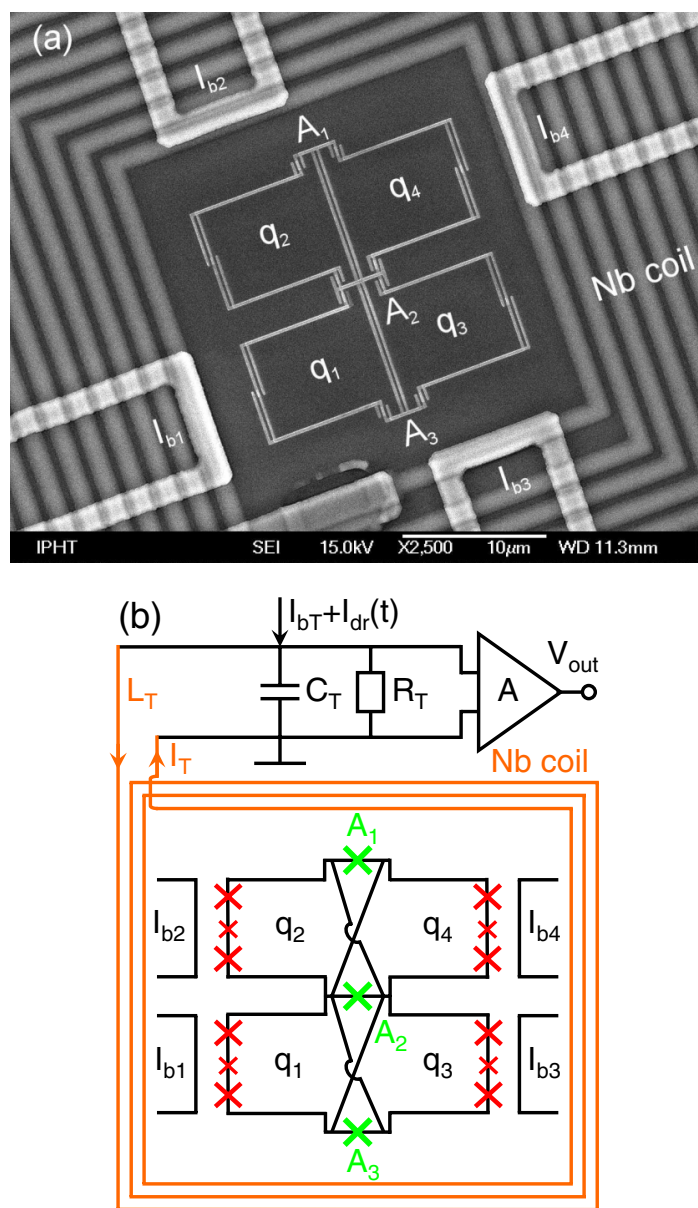

FIG. 1 (color online). (a) Electron micrograph of the sample. The central junctions $A_{1}-A_{3}$ couple the Al qubits $q_{1}-q_{4}$. The surrounding $\mathrm{Nb}$ coil is part of the $L C$ tank circuit, used for both measurement and global flux biasing. The $\mathrm{Nb}$ lines $I_{\mathrm{b} 1-\mathrm{b} 4}$ allow asymmetric bias tuning. (b) Schematic diagram. The driving current $I_{\mathrm{dr}}(t)$ is much smaller than the oscillations in $I_{\mathrm{T}}$ and only needs to compensate for the latter's losses.

where $L_{\mathrm{T}}$ and $Q_{\mathrm{T}}$ are the tank's inductance and quality factor, respectively, $\rho_{\mu}=e^{-E_{\mu} / T} / \sum_{\nu} e^{-E_{\nu} / T}$ is the Boltzmann factor of the eigenstate $|\mu\rangle$ of $H$ in (1), and with the matrix element

$$
R_{\mu \nu}=\sum_{i, j=1}^{4} \lambda_{i} \lambda_{j}\left\langle\mu\left|\sigma_{z}^{(i)}\right| \nu\right\rangle\left\langle\nu\left|\sigma_{z}^{(j)}\right| \mu\right\rangle
$$

$\lambda_{i}$ are tank-qubit coupling coefficients in $H_{\text {int }}=$ $I_{\mathrm{rf}} \sum_{i} \lambda_{i} \sigma_{z}^{(i)}$, and $I_{\mathrm{rf}}$ is the oscillating component of the tank current $I_{\mathrm{T}}=I_{\mathrm{bT}}+I_{\mathrm{rf}}(t)$. The term $i=j$ produces the IMT peak corresponding to qubit $i$. Cross terms $i \neq j$, on the other hand, appear only if $|\mu\rangle$ or $|\nu\rangle$ has nonzero entanglement between qubits $i$ and $j$ due to their interaction. For two weakly coupled qubits [4], the effect of interaction can be understood as a correction. The cross terms in $R_{\mu \nu}$ then change the shape of overlapping peaks at codegeneracies [i.e., when the $\epsilon_{i}$ in (1) are chosen such that both qubits flip simultaneously], which turn out to be

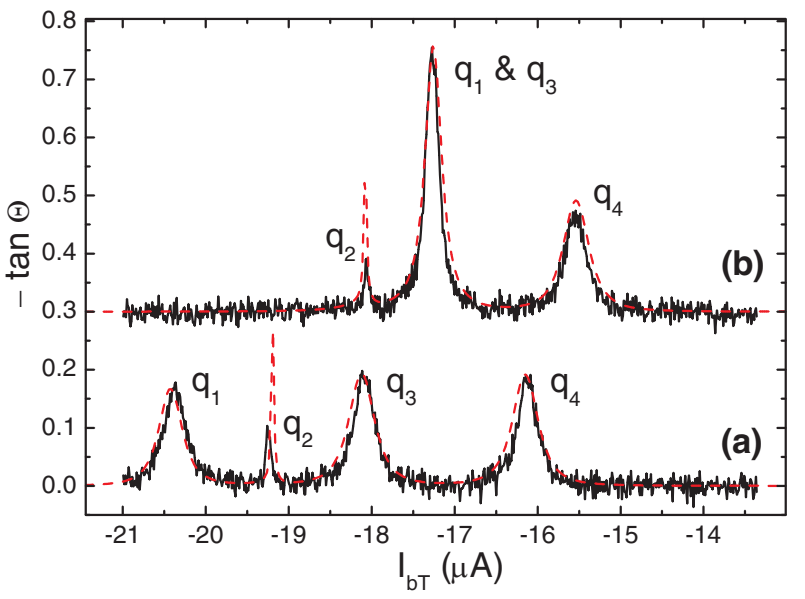

FIG. 2 (color online). (a) $-\tan \Theta\left(I_{\mathrm{bT}}\right)$ at $I_{\mathrm{b} 1}=700 \mu \mathrm{A}, I_{\mathrm{b} 2}=$ $10 \mu \mathrm{A}$, and $I_{\mathrm{b} 4}=250 \mu \mathrm{A}\left(I_{\mathrm{b} 3}=0\right.$ throughout $)$. The four qubit peaks do not overlap. (b) The same graph (vertically shifted for clarity) but at $I_{\mathrm{b} 1}=268 \mu \mathrm{A}$, where the peaks corresponding to $q_{1}$ and $q_{3}$ overlap. The height increase compared to the sum of the two nonoverlapping peaks is a signature of FM coupling. Dashed lines are theoretical fits. The fit for $q_{2}$ is poor because the relatively large tank amplitude washes out the experimental peak [13]; a better fit has been obtained for smaller amplitudes (not shown).

smaller (larger) than the sum of the two individual peaks for AFM (FM) coupling [16].

Here, the analysis needs to be extended both beyond two qubits and to stronger coupling (particularly $J>\Delta$ ), which qualitatively changes the locus of the IMT peaks, as can already be understood classically: neglecting the $\Delta_{i}$ in (1), the degeneracy points of the classical multiqubit flux states are seen to depend on the $J_{i j}$ [6]. One then proceeds by fitting the coefficients of $H$ in (1); see below.

Figure 1(a) shows the sample with four Al qubit loops inside a $\mathrm{Nb}$ pancake coil. The areas of the Al loops are $10 \times 7.5 \mu \mathrm{m}^{2}$. Two junctions in each qubit are typically $650 \times 150 \mathrm{~nm}^{2}$, while the third is $\sim 25 \%$ smaller [2]. The $\mathrm{Nb}$ coil has $L_{\mathrm{T}}=105 \mathrm{nH}$, and together with an external capacitance $C_{\mathrm{T}}=470 \mathrm{pF}$ forms a parallel tank circuit with $\omega_{\mathrm{T}} / 2 \pi=22.703 \mathrm{MHz}$ and $Q_{\mathrm{T}}=\omega_{\mathrm{T}} R_{\mathrm{T}} C_{\mathrm{T}}=400$, where $R_{\mathrm{T}}$ is the effective resistance. The $\mathrm{Al}$ loops were fabricated by $e$-beam lithography and conventional shadow evaporation, and the $\mathrm{Nb}$ coil by $e$-beam lithography and $\mathrm{CF}_{4}$ reactive-ion etching.

The qubits are coupled to each other both magnetically and via a shared junction, consisting of parallel junctions $A_{1}-A_{3}$ (Fig. 1). For a coupling junction with large critical current $I_{\mathrm{c}}$, and assuming identical qubits, $J=\hbar I_{\mathrm{p}}^{2} / 2 e I_{\mathrm{c}}[6]$. For our system, $I_{\mathrm{p}} \sim 250 \mathrm{nA}$. Because of the relative twist [6] between the qubit loops [Fig. 1(b)], the couplings $q_{1}-q_{3}$ and $q_{2}-q_{4}$ are FM $\left(J_{i j}<0\right)$, while the others are $\operatorname{AFM}\left(J_{i j}>0\right)$. The biases $\epsilon_{i}$ can be varied by adjusting the currents $I_{\mathrm{b} 1-\mathrm{b} 4}$ through the $\mathrm{Nb}$ wires, as well as the dc current $I_{\mathrm{bT}}$ through the tank coil. The qubit-coil mutual 

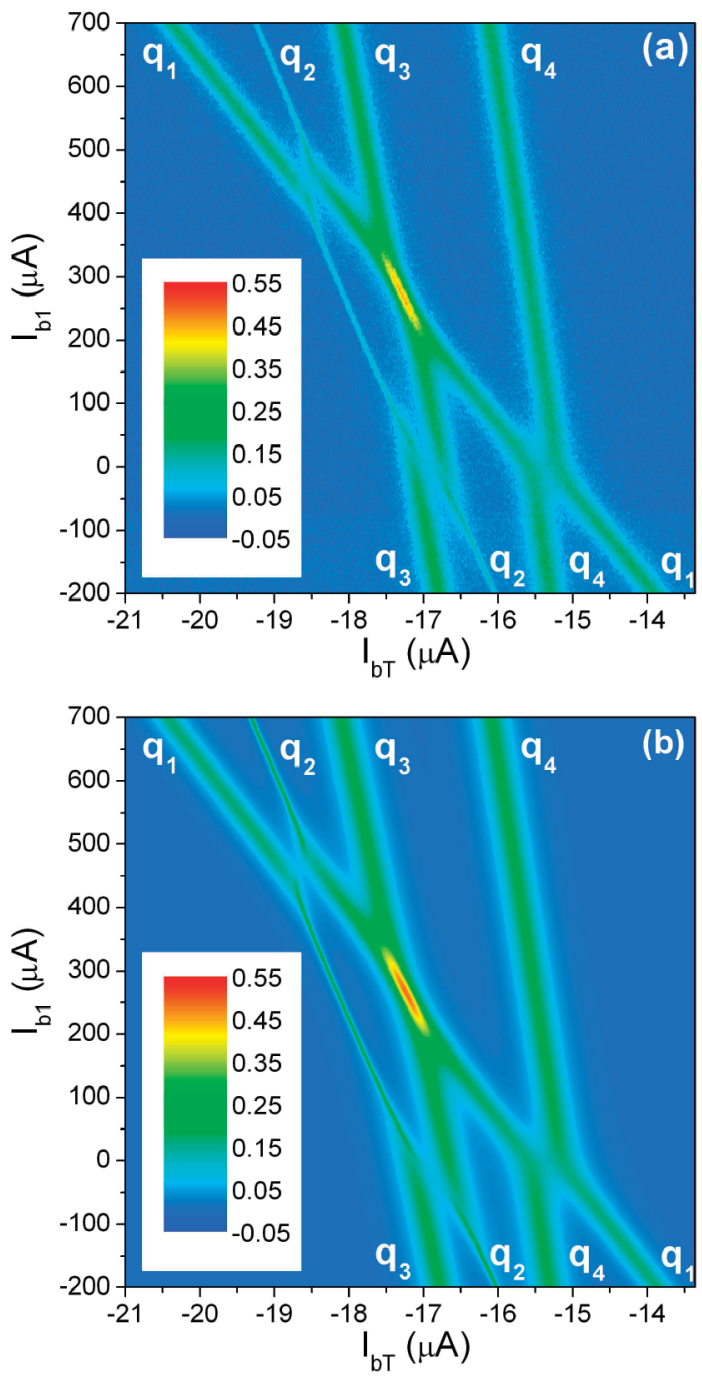

FIG. 3 (color). (a) $-\tan \Theta\left(I_{\mathrm{bT}}, I_{\mathrm{b} 1}\right)$ at $I_{\mathrm{b} 2}=10 \mu \mathrm{A}$ and $I_{\mathrm{b} 4}=$ $250 \mu \mathrm{A}$. The repulsion between the traces $\left(q_{1}, q_{2}\right),\left(q_{2}, q_{3}\right)$, and $\left(q_{1}, q_{4}\right)$ shows AFM coupling between the qubits in those pairs, while the merging of traces $\left(q_{1}, q_{3}\right)$ demonstrates FM coupling. The line widths are proportional to the tunneling amplitudes of the individual qubits. Since the qubits' sensitivity to $I_{\mathrm{b} 1}$ decreases in the order $q_{1}, q_{2}, q_{3}, q_{4}$ [cf. Figure 1(a)], the various slopes allow one to identify the trace belonging to each qubit. (b) Theoretical graph for the same parameters.

inductances follow from the flux periodicity as $M_{q 1, \mathrm{~T}} \approx$ $M_{q 3, \mathrm{~T}} \approx 38.2 \mathrm{pH}, M_{q 2, \mathrm{~T}}=38.6 \mathrm{pH}$, and $M_{q 4, \mathrm{~T}}=38.9 \mathrm{pH}$.

The sample was thermally anchored to the mixing chamber of a dilution refrigerator at a temperature $T_{\text {mix }} \approx$ $10 \mathrm{mK}$. In general the effective $T$ is affected by noise on external leads and so is larger than $T_{\text {mix }}$. Often, $T_{\text {mix }}$ alone is given, since $T$ is difficult to determine. One of the advantages of IMT is that it allows one to determine effective temperatures $[6,14]$. From the best theoretical fit we estimate $T \approx 70 \mathrm{mK}$, well within the expected range.

Figure 2 shows peaks in $-\tan \Theta$ as the overall flux is scanned by changing $I_{\mathrm{bT}}$. For most biases, four peaks can be distinguished [Fig. 2(a)], each corresponding to degen-
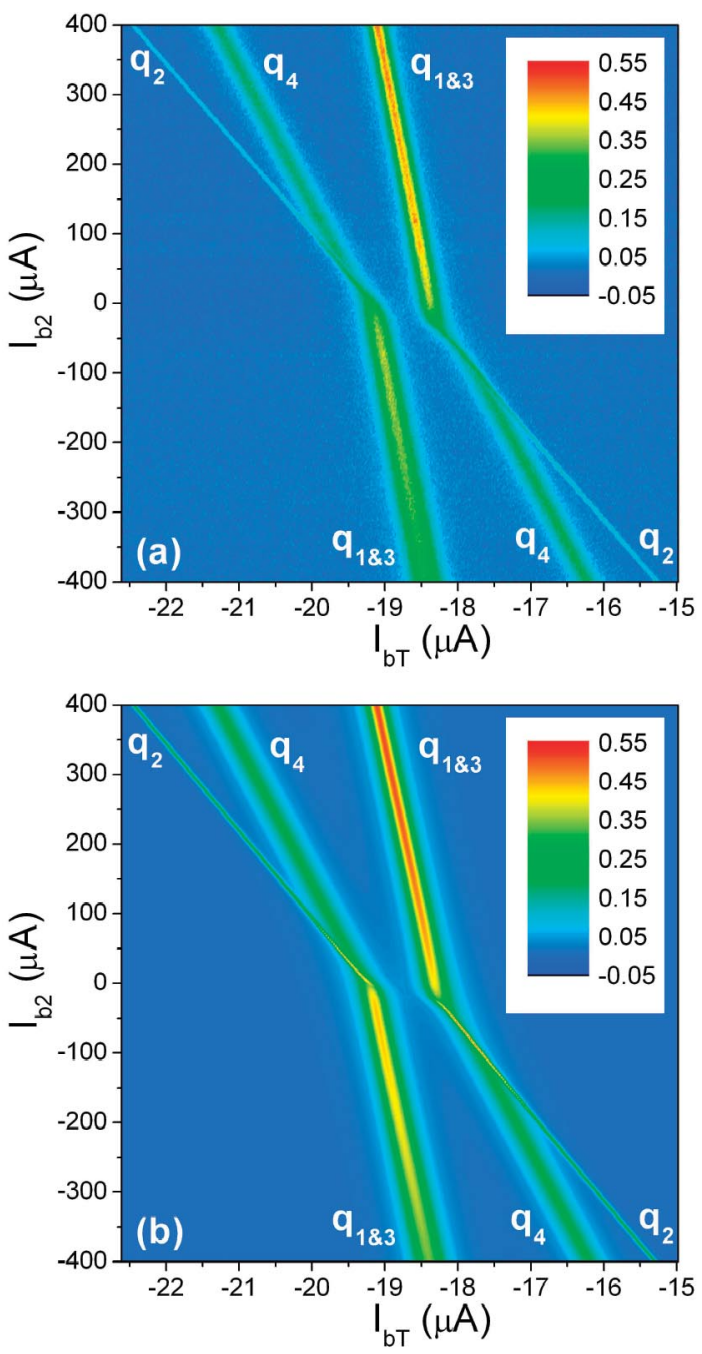

FIG. 4 (color). Same as in Fig. 3, but as a function of $I_{\mathrm{bT}}$ and $I_{\mathrm{b} 2}$, at $I_{\mathrm{b} 1}=-400 \mu \mathrm{A}$ and $I_{\mathrm{b} 4}=-50 \mu \mathrm{A}$. The skipped crossing at the center corresponds to four-qubit codegeneracy. Because of their FM coupling, the $q_{1}$ and $q_{3}$ traces merge over an extended region. The qubits' sensitivity to $I_{\mathrm{b} 2}$ decreases in the order $q_{2}, q_{4}, q_{1,3}$.

eracy in one of the qubits while the others provide a semiclassical static background field. The narrow peak for $q_{2}$ indicates a small $\Delta_{2}(\sim 12 \mathrm{mK})$, and incidentally confirms that the width of the other traces is not resolution limited. When $q_{1}$ and $q_{3}$ are codegenerate [Fig. 2(b)], the resulting peak exceeds the sum of their individual peaks, indicating strong FM coupling as mentioned above (this is also true for $q_{2}$ and $q_{4}$ ).

Figure 3(a) plots $-\tan \Theta\left(I_{\mathrm{bT}}, I_{\mathrm{b} 1}\right)$. The four traces are IMT peaks, each corresponding to a single-qubit anticrossing. Since $I_{\mathrm{bT}}$ biases all qubits almost equally, the trace with the greatest sensitivity to $I_{\mathrm{b} 1}$ can be ascribed to $q_{1}$ etc. Combining several such cross-sections of the total IMT response, one reconstructs the biasing coefficients in $\Phi_{i}^{\mathrm{x}}=$ $M_{q i, \mathrm{~T}} I_{\mathrm{bT}}+\sum_{j} M_{q i, \mathrm{~b} j}^{\prime} I_{\mathrm{b} j}$. The centers of the traces mark the boundaries of the classical stability diagram. Each region 
between the traces corresponds to a different (minimumpotential) flux state of the qubits [17]. The shifts of the traces after each (skipped) crossing depend on the $J_{i j}$. The peak shapes, on the other hand, carry information about the $\Delta_{i}$. As expected, the traces corresponding to two qubits with AFM (FM) coupling repel (attract) each other, and in the FM case merge over a certain distance. Fitting the data to Eq. (1) yields $\Delta_{1}=147, \Delta_{2}=12, \Delta_{3}=163$, $\Delta_{4}=165, \quad$ and $\quad J_{12}=J_{34}=163, \quad J_{14}=J_{23}=155$, $J_{13}=J_{24}=-62$ (all in $\mathrm{mK}$ ), and

$$
M^{\prime}=\left(\begin{array}{rrrr}
247 & 71 & \cdot & 68 \\
143 & 309 & \cdot & 84 \\
70 & 87 & \cdot & 182 \\
50 & 195 & \cdot & 355
\end{array}\right) \mathrm{fH},
$$

where the missing entries correspond to $I_{\mathrm{b} 3}$ which was never used. The theory [Fig. 3(b)] accurately describes the system's behavior over the whole parameter space, especially at the codegeneracies.

Figure 4(a) shows $-\tan \Theta\left(I_{\mathrm{bT}}, I_{\mathrm{b} 2}\right)$ for a case when all four qubits become degenerate simultaneously in the figure's center, where they produce two repelling peaks. From $H$ in (1) one infers that, between those peaks, the two lowest eigenstates are close to superpositions of $|\uparrow \downarrow \uparrow \downarrow\rangle$ and $|\downarrow \uparrow \downarrow \uparrow\rangle$, for which all the coupling energies are negative; at codegeneracy, the superpositions become (anti)symmetric [18]. From the viewpoint of AQC, it is encouraging that the ground state of $H$ thus is globally entangled [19]; for a quantification, see Ref. [20]. With all parameters already determined from Fig. 3 and a few other cross-sections like it, the theoretical comparison in Fig. 4(b) (and many other nontrivial cross-sections) can be regarded as having no free parameters, making the agreement remarkable.

Complete agreement between experiment and theory was also obtained for a second sample, with $\Delta_{1}=\Delta_{2}=60, \Delta_{3}=130, \Delta_{4}=110, J_{12}=J_{23}=300$, $J_{14}=J_{34}=330$, and $J_{13}=J_{24}=-90$ (all in $\mathrm{mK}$ ). However, the lowest effective temperature attained was only $\sim 300 \mathrm{mK}$.

In conclusion, ferromagnetic and antiferromagnetic couplings between four 3JJ flux qubits have been realized simultaneously with shared Josephson junctions, with a coupling strength significantly exceeding the inductive one. (Incidentally, the results also show that direct galvanic coupling can be used for more general circuits than the linear arrays considered in Ref. [21].) The data fully agree with a quantum-mechanical description to the experimental accuracy. Currently, quasiequilibrium impedance measurement already provides valuable information complementary to state readout, notably the determination of the Hamiltonian. With a faster and possibly qubit-selective detection method, the circuit used looks very promising for studying adiabatic bias manipulations.

M. G. was supported by Grants No. VEGA 1/2011/05 and No. APVT-51-016604, E. I. by the RSFQubit project, and A. Z. by the NSERC Discovery Grants Program. We thank A. J. Berkley, D. A. Lidar, and M. F. H. Steininger for discussions.

*Electronic address: ilichev@ipht-jena.de

${ }^{\dagger}$ Electronic address: alec@dwavesys.com

Electronic address: zagoskin@physics.ubc.ca

[1] Y. Nakamura, Yu. A. Pashkin, and J.S. Tsai, Nature (London) 398, 786 (1999); D. Vion et al., Science 296, 886 (2002); T. Yamamoto, Yu. A. Pashkin, O. Astafiev, Y. Nakamura, and J. S. Tsai, Nature (London) 425, 941 (2003).

[2] J. E. Mooij et al., Science 285, 1036 (1999).

[3] E. Il'ichev et al., Phys. Rev. Lett. 91, 097906 (2003); P. Bertet et al., Phys. Rev. Lett. 95, 257002 (2005).

[4] A. Izmalkov et al., Phys. Rev. Lett. 93, 037003 (2004).

[5] L. S. Levitov, T. P. Orlando, J. B. Majer, and J. E. Mooij, cond-mat/0108266.

[6] M. Grajcar et al., Phys. Rev. B 72, 020503(R) (2005).

[7] For other designs with FM coupled flux qubits, see J. Q. You, Y. Nakamura, and F. Nori, Phys. Rev. B 71, 024532 (2005) and Ref. [6].

[8] F. Barahona, J. Phys. A 15, 3241 (1982).

[9] E. Farhi, J. Goldstone, S. Gutmann, and M. Sipser, quant-ph/0001106; W. M. Kaminsky and S. Lloyd, quant-ph/0211152;W. M. Kaminsky, S. Lloyd, and T. P. Orlando, quant-ph/0403090.

[10] M. Grajcar, A. Izmalkov, and E. Il'ichev, Phys. Rev. B 71, 144501 (2005).

[11] I. Chiorescu, Y. Nakamura, C. J. P. M. Harmans, and J. E. Mooij, Science 299, 1869 (2003); T. P. Orlando et al., Phys. Rev. B 60, 15398 (1999); A. Lupaşcu, C. J. M. Verwijs, R. N. Schouten, C. J.P. M. Harmans, and J.E. Mooij, Phys. Rev. Lett. 93, 177006 (2004).

[12] A. Maassen van den Brink, Phys. Rev. B 71, 064503 (2005).

[13] Ya. S. Greenberg et al., Phys. Rev. B 66, 214525 (2002).

[14] M. Grajcar et al., Phys. Rev. B 69, 060501(R) (2004).

[15] A. Yu. Smirnov, cond-mat/0312635.

[16] This is plausible: for stronger AFM coupling the codegeneracy develops into an $|\uparrow \downarrow\rangle \leftrightarrow|\downarrow \uparrow\rangle$ anticrossing line with no net flux change, hence no IMT signature at all in the symmetric case [6]. On the other hand, for strong FM coupling, an $|\uparrow \uparrow\rangle \leftrightarrow|\downarrow\rangle\rangle$ line develops, i.e., a sharp secondorder anticrossing with a large IMT response.

[17] The stability diagram is completed by connecting the opposing corners where traces corresponding to skipped crossings meet. These invisible lines correspond to the $|\uparrow \downarrow\rangle \leftrightarrow|\downarrow \uparrow\rangle$ anticrossings.

[18] However, the minimum energy gap $E_{1}-E_{0} \approx 8 \mathrm{mK}$ between $\left|\mu_{1}\right\rangle$ and $\left|\mu_{0}\right\rangle$ (due to fourth-order tunneling in perturbative language) was less than the thermal energy, partly because of the small $\Delta_{2}$. Therefore, we do not claim selectivity between these GHZ-type states.

[19] G. Vidal, Phys. Rev. Lett. 91, 147902 (2003).

[20] P. J. Love et al. (to be published).

[21] J.R. Butcher, M.Sc. thesis, Delft University of Technology, 2002. 